\title{
On Boussinesq models of constant depth
}

\author{
Jin E. Zhang ${ }^{\text {a) }}$ \\ Department of FINA, Hong Kong University of Science and Technology, Clear Water Bay, \\ Kowloon, Hong Kong \\ and Department of Engineering Science, California Institute of Technology, Pasadena, \\ California 91125
}

Chunli Chen

Department of Mathematics, Shanghai Jiao Tong University, Shanghai 200030, People's Republic of China

Yishen Li

Department of Mathematics, University of Science and Technology of China, Hefei 230026, People's Republic of China

(Received 11 August 2003; accepted 20 January 2004; published online 2 April 2004)

The mathematical properties, such as integrability, symmetries and multiple solitary wave solutions of Boussinesq models of constant depth are studied. An integrable modified Boussinesq model has been identified. () 2004 American Institute of Physics. [DOI: 10.1063/1.1688323]

\section{INTRODUCTION}

When modeling water waves, one often assume that water is incompressible and inviscid and the flow is irrotational. The evolution of nonlinear water waves is governed by the Euler equations. There are two important parameters associated with long waves. One is the ratio of amplitude to depth, represented by $\alpha$, and the other one is the ratio of depth to wavelength, represented by $\epsilon$. Under the assumption that

$$
\alpha \ll 1, \quad \epsilon \ll 1, \quad \text { and } \alpha=O\left(\epsilon^{2}\right),
$$

one may derive the original Boussinesq equations

$$
\begin{aligned}
& \zeta_{t}+\nabla \cdot[(1+\zeta) \overline{\boldsymbol{u}}]=0, \\
& \overline{\boldsymbol{u}}_{t}+\overline{\boldsymbol{u}} \cdot \nabla \overline{\boldsymbol{u}}+\nabla \zeta=-\nabla p_{a}+\frac{1}{3} \nabla^{2} \overline{\boldsymbol{u}}_{t},
\end{aligned}
$$

from the Euler equations by using a perturbation scheme. The Boussinesq equations can be used to model the propagation of nonlinear long waves on a layer of water with constant depth, normalized to be 1 . Here, $\zeta$ is wave elevation; $\overline{\boldsymbol{u}}$ is the horizontal projection of depth-mean velocity; $p_{a}$ is ambient surface gauge pressure.

Peregrine $^{1}$ derived a Boussinesq model

$$
\begin{aligned}
\zeta_{t}+\nabla \cdot[(h+\zeta) \overline{\boldsymbol{u}}] & =0, \\
\overline{\boldsymbol{u}}_{t}+\overline{\boldsymbol{u}} \cdot \nabla \overline{\boldsymbol{u}}+\nabla \zeta= & -\nabla p_{a}+\frac{h}{2} \frac{\partial}{\partial t} \nabla[\nabla \cdot(h \overline{\boldsymbol{u}})] \\
& -\frac{h^{2}}{6} \frac{\partial}{\partial t} \nabla(\nabla \cdot \overline{\boldsymbol{u}}),
\end{aligned}
$$

for long waves in water of depth varying in space, i.e., $h$ $=h(x, y)$, and used the model to study wave evolution near a beach.

$\mathrm{Wu}^{2}$ derived a generalized Boussinesq $(\mathrm{gB})$ model

$$
\zeta_{t}+\nabla \cdot[(h+\zeta) \bar{u}]=-h_{t},
$$

\footnotetext{
a) Author to whom correspondence should be addressed. Telephone: (852) 2358 8367; fax: (852) 2358 1749. Electronic mail: jinzhang@ust.hk
}

$$
\begin{aligned}
\overline{\boldsymbol{u}}_{t}+\overline{\boldsymbol{u}} \cdot \nabla \overline{\boldsymbol{u}}+\nabla \zeta= & -\nabla p_{a}+\frac{h}{2} \frac{\partial}{\partial t} \nabla\left[h_{t}+\nabla \cdot(h \overline{\boldsymbol{u}})\right] \\
& -\frac{h^{2}}{6} \frac{\partial}{\partial t} \nabla^{2} \overline{\boldsymbol{u}}
\end{aligned}
$$

which included depth variations in space and time, i.e., $h$ $=h(x, y, t)$. Wu's gB model can be used to study not only long wave propagation over variable depth (as Peregrine's standard Boussinesq model) but also the forced generation of long waves by external moving disturbances. ${ }^{3} \mathrm{Wu}^{2}$ also rewrote the Boussinesq model in terms of wave elevation and velocity potential in order to reduce the number of unknowns in the equations. This new form has proved to be more efficient for numerical simulations and has been widely applied. For example, Wu's gB model is used to study the threedimensional interaction between a solitary wave and a vertical cylinder, ${ }^{4}$ a breakwater. ${ }^{5}$ It is also used to study the turning of a solitary wave in a curved channel ${ }^{6}$ and run-up of ocean waves on beaches. ${ }^{7-9}$

Nwogu ${ }^{10}$ derived an extended Boussinesq model by introducing a velocity, $\boldsymbol{u}$, at an arbitrary depth, $z_{\alpha}(x, y)\left[z_{\alpha}\right.$ $=0$ is on the surface, $z_{\alpha}=-h(x, y)$ is at the bottom],

$$
\begin{aligned}
\zeta_{t}+\nabla \cdot[(h+\zeta) \boldsymbol{u}]+\nabla \cdot\left\{\left(\frac{z_{\alpha}^{2}}{2}-\frac{h^{2}}{6}\right) h \nabla(\nabla \cdot \boldsymbol{u})\right. \\
\left.\quad+\left(z_{\alpha}+\frac{h}{2}\right) h \nabla[\nabla \cdot(h \boldsymbol{u})]\right\}=0, \\
\boldsymbol{u}_{t}+\boldsymbol{u} \cdot \nabla \boldsymbol{u}+\nabla \zeta+z_{\alpha}\left\{\frac{z_{\alpha}}{2} \nabla\left(\nabla \cdot \boldsymbol{u}_{t}\right)+\nabla\left[\nabla \cdot\left(h \boldsymbol{u}_{t}\right)\right]\right\}=-\nabla p_{a} .
\end{aligned}
$$

He found that if the velocity at depth $z_{\alpha}=-0.531(h=1)$ is used, the dispersion relation of the Boussinesq model can be improved to be closer to the dispersion relation based on the potential theory. This helps to make the extended Boussinesq model applicable to broader range of water depth, i.e., not only for shallow water but also for deeper water. This has 
been justified numerically by Wei and Kirby, ${ }^{11}$ and Wei et al. ${ }^{12}$ with a fully nonlinear Boussinesq model.

The Boussinesq models (1) and (2) has been extended to higher-order by $\mathrm{Wu}$ and Zhang ${ }^{13}$ for constant water depth $h$ $=1$. They are presented here in three different velocity systems:

(A) the $\{\zeta, \overline{\boldsymbol{u}}\}$ system-the depth-mean velocity basis

$$
\begin{aligned}
\zeta_{t}+\nabla \cdot[(1+\zeta) \overline{\boldsymbol{u}}] & =0, \\
\overline{\boldsymbol{u}}_{t}+\overline{\boldsymbol{u}} \cdot \nabla \overline{\boldsymbol{u}}+\nabla \zeta= & -\nabla p_{a}+\frac{1}{3}(1+\zeta)^{2} \nabla^{2} \overline{\boldsymbol{u}}_{t}+\frac{1}{45} \nabla^{4} \overline{\boldsymbol{u}}_{t} \\
& +(\nabla \zeta) \nabla \cdot \overline{\boldsymbol{u}}_{t}+\frac{1}{3} \nabla\left[\overline{\boldsymbol{u}} \cdot \nabla^{2} \overline{\boldsymbol{u}}-(\nabla \cdot \overline{\boldsymbol{u}})^{2}\right]
\end{aligned}
$$

(B) the $\left\{\zeta, \boldsymbol{u}_{0}\right\}$ system-the bottom variable basis

$$
\begin{aligned}
\zeta_{t}+\nabla \cdot & {\left[(1+\zeta) \boldsymbol{u}_{0}\right]=\nabla \cdot\left[\frac{1}{6}(1+\zeta)^{3} \nabla^{2} \boldsymbol{u}_{0}-\frac{1}{5 !} \nabla^{4} \boldsymbol{u}_{0}\right], } \\
\boldsymbol{u}_{0 t}+\boldsymbol{u}_{0} \cdot \nabla \boldsymbol{u}_{0}+\nabla \zeta & \\
= & -\nabla p_{a}+\frac{1}{2}(1+\zeta)^{2} \nabla^{2} \boldsymbol{u}_{0 t}-\frac{1}{4 !} \nabla^{4} \boldsymbol{u}_{0 t}+(\nabla \zeta) \nabla \cdot \boldsymbol{u}_{0 t} \\
& +\frac{1}{2} \nabla\left[\boldsymbol{u}_{0} \cdot \nabla^{2} \boldsymbol{u}_{0}-\left(\nabla \cdot \boldsymbol{u}_{0}\right)^{2}\right] ;
\end{aligned}
$$

(C) the $\{\zeta, \hat{\boldsymbol{u}}\}$ system-the surface variable basis

$$
\begin{aligned}
& \zeta_{t}+\nabla \cdot[(1+\zeta) \hat{\boldsymbol{u}}]=-\nabla \cdot\left[\frac{1}{3}(1+\zeta)^{3} \nabla^{2} \hat{\boldsymbol{u}}+\frac{2}{15} \nabla^{4} \hat{\boldsymbol{u}}\right], \\
& \hat{\boldsymbol{u}}_{t}+\hat{\boldsymbol{u}} \cdot \nabla \hat{\boldsymbol{u}}+\nabla \zeta=-\nabla p_{a}+(\nabla \zeta) \nabla \cdot \hat{\boldsymbol{u}}_{t} .
\end{aligned}
$$

A leading order reduced version of system (C)

$$
\begin{aligned}
& \zeta_{t}+\nabla \cdot[(1+\zeta) \hat{\boldsymbol{u}}]=-\frac{1}{3} \nabla \cdot \nabla^{2} \hat{\boldsymbol{u}}, \\
& \hat{\boldsymbol{u}}_{t}+\hat{\boldsymbol{u}} \cdot \nabla \hat{\boldsymbol{u}}+\nabla \zeta=0,
\end{aligned}
$$

is regarded as $\mathrm{Wu}$-Zhang equation and studied with a Painléve analysis approach by Ref. 14. Its Lie symmetry analysis and some new exact solutions are provided recently by Ref. 15 .

If the water wave is uniform along $y$ direction, all the dependent variables are functions of $x$ and $t$ only, the leading order Boussinesq models are reduced to

(a) the $\{\zeta, \bar{u}\}$ system-

$$
\begin{aligned}
& \zeta_{t}+[(1+\zeta) \bar{u}]_{x}=0, \\
& \bar{u}_{t}+\bar{u} \bar{u}_{x}+\zeta_{x}=\frac{1}{3} \bar{u}_{x x t}
\end{aligned}
$$

(b) the $\left\{\zeta, u_{0}\right\}$ system-

$$
\begin{aligned}
& \zeta_{t}+\left[(1+\zeta) u_{0}\right]_{x}=\frac{1}{6} u_{0 x x x}, \\
& u_{0 t}+u_{0} u_{0 x}+\zeta_{x}=\frac{1}{2} u_{0 x x t} ;
\end{aligned}
$$

(c) the $\{\zeta, \hat{u}\}$ system-

$$
\begin{aligned}
& \zeta_{t}+[(1+\zeta) \hat{u}]_{x}=-\frac{1}{3} \hat{u}_{x x x}, \\
& \hat{u}_{t}+\hat{u} \hat{u}_{x}+\zeta_{x}=0 .
\end{aligned}
$$

We may write the three sets of Boussinesq model in Nwogu's ${ }^{10}$ unified form as follows:

(d) A unified 1+1 leading order Boussinesq model,

$$
\begin{aligned}
& \zeta_{t}+[(1+\zeta) u]_{x}=a u_{x x x}, \\
& u_{t}+u u_{x}+\zeta_{x}=b u_{x x t},
\end{aligned}
$$

where

$$
a=-\frac{1}{2}\left(\theta^{2}-\frac{1}{3}\right), \quad b=\frac{1}{2}\left(1-\theta^{2}\right),
$$

and the velocity $u$ is taken at depth $\theta=z_{\alpha}+1(\theta=0$ is at the bottom, $\theta=1$ is on the surface). Depth-mean system is equivalent to a system at a water depth $\theta=1 / \sqrt{3}$.

Since the Boussinesq model is very useful in coastal and civil engineering, the mathematical properties such as integrability, symmetries and solitary wave (soliton) solutions of the system are important to us. That is the focus of this paper.

We will study the Painlevé property of the Boussinesq model in Sec. II, and Lie symmetries in Sec. III. The single solitary wave solution will be studied in Sec. IV. In Sec. V, we will present an approximate analytical multi-soliton solution of the Boussinesq model by using the exact multi-soliton solution of system (c) developed recently by Zhang and $\mathrm{Li}^{16-20}$ In Sec. VI, we discuss the integrability of the Boussinesq model, and present an integrable modified Boussinesq model that is a variation of system (c). It turns out that the approximate solutions presented in Sec. V are exact solutions of the modified Boussinesq model. Finally we conclude the paper in Sec. VII.

\section{PAINLEVÉ ANALYSIS}

We begin our study with the Painlevé properties of the Boussinesq model.

The Painlevé property of a system of nonlinear differential equations is defined as that all the solutions of the system are free of the essential and branch movable singularities around an arbitrary (both characteristic and noncharacteristic) singular manifold. An analysis that checks whether a given nonlinear differential equation has Painlevé property is generally termed Painlevé analysis. The Painlevé analysis developed by Weiss, Tabor, and Carnevale ${ }^{21}$ plays an important role in soliton theory. It can be used to identify the Painlevé-integrability of a system. It can also be used to find some other integrable properties such as the Bäcklund transformation, Lax pair and Schwarzian form, etc. Furthermore, even if a model is not Painlevé-integrable, the Painlevé analysis can still be used to find an exact explicit solution of the system in some particular forms.

The system (c), i.e., system (d) with $b=0$, is known to be integrable and equivalent to Broer-Kaup (BK) and a member of Ablowitz-Kaup-Newell-Segur (AKNS) system. Its Painlevé property has been studied by Sachs. ${ }^{22}$ Here we only check the Painlevé property of system (d) with $b \neq 0$.

According to the definition of the Painlevé property, a general solution of the Boussinesq model, i.e., system (d), should have the following expansion around an arbitrary singular manifold $\phi \equiv \phi(x, y, t)=0$

$$
\zeta=\sum_{j=0}^{\infty} \zeta_{j} \phi^{j+\alpha}, \quad u=\sum_{j=0}^{\infty} u_{j} \phi^{j+\beta},
$$

where $\alpha$ and $\beta$ are two negative integers. Furthermore, if the model has the Painlevé property, there should be at least one primary branch, i.e., one possible choice of $\left\{\alpha, \beta, \zeta_{0}, u_{0}\right\}$, 
such that enough arbitrary functions can be included in the Painlevé expansions. For system (d), we need six arbitrary functions, including $\phi$ and five $\zeta_{j}$ and $u_{j}$ s.

By using the standard leading order analysis, i.e., substituting $\zeta=\zeta_{0} \phi^{\alpha}, u=u_{0} \phi^{\beta}$ into the Boussinesq model and balancing the leading terms, we can find that if $a \neq 0$, there is only one possible choice

$$
\alpha=-2, \quad \beta=-2, \quad \zeta_{0}=6 a \phi_{x}^{2}, \quad u_{0}=12 b \phi_{x} \phi_{t} .
$$

If $a=0$, it is impossible to find suitable negative integers for $\alpha$ and $\beta$. Substituting the above expansion (17) together with (18) into system (d) and setting the coefficient of the power $\phi^{k}$ vanish for all $k$, we have the following recursive formula for the expansion functions $\zeta_{j}$ and $u_{j}$ :

$$
\begin{aligned}
& a \phi_{x} j(j-4)(j-5) u_{j}-12 b \phi_{t}(j-4) \zeta_{j}=F_{1 j}, \\
& (j+1)(j-4)(j-6) u_{j}=F_{2 j},
\end{aligned}
$$

where $F_{1 j}$ and $F_{2 j}$ are functions of $\zeta_{0}, \zeta_{1}, \ldots, \zeta_{j-1}, u_{0}$, $u_{1}, \ldots, u_{j-1}$ and the derivatives of $\phi$ only. From the recursive relations (19) and (20), we know that all the functions $\zeta_{j}$ and $u_{j}$ are determined except those related to the resonance points, which are determined by the coefficient determinant

$$
\begin{gathered}
\left|\begin{array}{cc}
a \phi_{x} j(j-4)(j-5) & -12 b \phi_{t}(j-4) \\
(j+1)(j-4)(j-6) & 0
\end{array}\right| \\
\quad=12 b \phi_{t}(j+1)(j-4)^{2}(j-6)=0,
\end{gathered}
$$

to be $j=-1,4$, and 6 . Checking the resonance conditions, we know that two facts destroy the Painlevé integrability of the Boussinesq model with $b \neq 0$. The first one is that there is no primary branch at all because the both equations of the Boussinesq model are third order, so the primary branch should have six resonant points such that six arbitrary functions enter into the general Painlevé expansion. The second fact is that the resonance conditions at $j=4$ and $j=6$ are not satisfied. The lack of the primary branch of the model means that some kind of the logarithmic branch and/or essential singularities around the singular manifold $\phi=0$ may appear for the Boussinesq model with $b \neq 0$. In fact, substituting $\zeta$ $=\zeta_{0} \phi^{\alpha}, u=u_{0} \phi^{\beta}$ into the Boussinesq model, we can find that there exists a possible noncompletely negative choice for constants $\alpha$ and $\beta$ :

$$
\alpha=0, \quad \beta=-2,
$$

for both cases of $a \neq 0$ and $a=0$ ! The allowance of the choice $\alpha=0$ means that the logarithmic term $\ln \phi$ should be included in the expansion of the function $u$ to balance the leading order terms of the first equation of system (d).

Summarizing the above analysis, we conclude that the Boussinesq model with $b \neq 0$ has no Painlevé property. In other words, the Boussinesq model is not Painlevéintegrable.

\section{LIE SYMMETRY ANALYSIS}

\section{A. Lie point symmetries}

In this section, we perform Lie symmetry analysis for the Boussinesq model. Let us consider a one-parameter Lie group of infinitesimal transformation

$$
\begin{array}{ll}
x \rightarrow x+\epsilon X(x, t, \zeta, u), & t \rightarrow t+\epsilon T(x, t, \zeta, u), \\
\zeta \rightarrow \zeta+\epsilon Z(x, t, \zeta, u), & u \rightarrow u+\epsilon U(x, t, \zeta, u),
\end{array}
$$

where $\epsilon$ is a small parameter, $\epsilon \ll 1$. The vector field associated with the above group of transformations can be written as

$$
\underline{v}=X \frac{\partial}{\partial x}+T \frac{\partial}{\partial t}+Z \frac{\partial}{\partial \zeta}+U \frac{\partial}{\partial u}
$$

The invariance of the system (d) under the infinitesimal transformations (23) leads to the expressions for the functions $X, T, Z$, and $U$ with a certain form. Throughout this paper we use the computer program MAPLE to obtain the Lie symmetries. The symmetries are listed below for three different cases:

Case 1: $a=0, b \neq 0$, system (a)_depth-mean velocity

$X=c_{1}, \quad T=c_{3} t+c_{2}, \quad Z=-2 c_{3}(1+\zeta), \quad U=-c_{3} u$,

where $c_{i},(i=1,2,3)$ are arbitrary constants. The presence of these arbitrary constants leads to a Lie algebra of symmetries. We can write a general element of the algebra as

$$
\underline{v}=\underline{v}_{1} c_{1}+\underline{v}_{2} c_{2}+\underline{v}_{3} c_{3},
$$

where

$$
\underline{v}_{1}=\frac{\partial}{\partial x}, \quad \underline{v}_{2}=\frac{\partial}{\partial t}, \quad \underline{v}_{3}=t \frac{\partial}{\partial t}-2(1+\zeta) \frac{\partial}{\partial \zeta}-u \frac{\partial}{\partial u} .
$$

The associated Lie algebra between these vector fields are $\left[\underline{v}_{i}, \underline{v}_{j}\right]=0,(i, j=1,2,3)$. The corresponding one-parameter group of symmetries of Boussinesq model are then

$$
\begin{aligned}
& G_{1}:(x, t, \zeta, u) \mapsto(x+\epsilon, t, \zeta, u), \\
& G_{2}:(x, t, \zeta, u) \mapsto(x, t+\epsilon, \zeta, u), \\
& G_{3}:(x, t, \zeta, u) \mapsto\left(x, t e^{\epsilon},(1+\zeta) e^{-2 \epsilon}-1, u e^{-\epsilon}\right) .
\end{aligned}
$$

The point transformation

$$
G_{i}:(x, t, \zeta, u) \mapsto(\xi, \eta, P, Q),
$$

means that if $\zeta(x, t), u(x, t)$ is a solution of Boussinesq model, then $P(\xi, \eta), Q(\xi, \eta)$ is a solution of the same Boussinesq model with $\xi$ and $\eta$ as independent variables.

Case 2: $a \neq 0, b \neq 0$, system (b) - bottom velocity

$$
X=c_{1}, \quad T=c_{2},
$$

where $c_{1}$ and $c_{2}$ are arbitrary constants. The general element of the algebra is

$$
\underline{v}=c_{1} \frac{\partial}{\partial x}+c_{2} \frac{\partial}{\partial t} \text {. }
$$


The corresponding one-parameter group of symmetries are two translations: Space (27) and time (28).

Case 3: $a \neq 0, b=0$, system (c)—surface velocity

$$
\begin{aligned}
& X=\frac{c_{4}}{2} x+c_{3} t+c_{1}, \quad T=c_{4} t+c_{2}, \\
& Z=-c_{4}(1+\zeta), \quad U=-\frac{c_{4}}{2} u+c_{3},
\end{aligned}
$$

where $c_{i},(i=1,2,3,4)$ are arbitrary constants. A general element of Lie algebra can be written as

$$
\underline{v}=\underline{v}_{1} c_{1}+\underline{v}_{2} c_{2}+\underline{v}_{3} c_{3}+\underline{v}_{4} c_{4},
$$

where

$$
\begin{aligned}
& \underline{v}_{1}=\frac{\partial}{\partial x}, \quad \underline{v}_{2}=\frac{\partial}{\partial t}, \quad \underline{v}_{3}=t \frac{\partial}{\partial x}+\frac{\partial}{\partial u}, \\
& \underline{v}_{4}=\frac{x}{2} \frac{\partial}{\partial x}+t \frac{\partial}{\partial t}-(1+\zeta) \frac{\partial}{\partial \zeta}-\frac{u}{2} \frac{\partial}{\partial u} .
\end{aligned}
$$

The associated Lie algebra between these vector fields are

$$
\begin{aligned}
& {\left[\underline{v}_{1}, \underline{v}_{4}\right]=\frac{\underline{v}_{1}}{2}, \quad\left[\underline{v}_{2}, \underline{v}_{3}\right]=\underline{v}_{1},} \\
& {\left[\underline{v}_{2}, \underline{v}_{4}\right]=\underline{v}_{2}, \quad\left[\underline{v}_{3}, \underline{v}_{4}\right]=-\frac{\underline{v}_{3}}{2},}
\end{aligned}
$$

and other $\left[\underline{v}_{i}, \underline{v}_{j}\right]=0$. The corresponding one-parameter group of symmetries of the Boussinesq model are

$$
\begin{aligned}
& G_{1}:(x, t, \zeta, u) \mapsto(x+\epsilon, t, \zeta, u), \\
& G_{2}:(x, t, \zeta, u) \mapsto(x, t+\epsilon, \zeta, u), \\
& G_{3}:(x, t, \zeta, u) \mapsto(x+t \epsilon, t, \zeta, u+\epsilon), \\
& G_{4}:(x, t, \zeta, u) \mapsto\left(x e^{\epsilon}, t e^{2 \epsilon},(1+\zeta) e^{-2 \epsilon}-1, u e^{-\epsilon}\right) .
\end{aligned}
$$

System (c) is integrable and has infinite number of symmetries. With the Lie symmetry analysis, we can only obtain four symmetries presented here. In order to obtain more symmetries of the system, one has to use other methods, such as the method of recursive operators.

\section{B. Similarity reductions}

After determining the infinitesimal generators, the similarity variables can be found by solving the characteristic equations see, e.g., Olver ${ }^{23}$

$$
\frac{d x}{X}=\frac{d t}{T}=\frac{d \zeta}{Z}=\frac{d u}{U} .
$$

Case 1: $a=0, b \neq 0$.

The generator $\underline{v}_{1}$ has an invariance

$$
\xi=t, \quad P=\zeta, \quad Q=u .
$$

Under this transformation, the Boussinesq model is reduced to a system of ordinary differential equation (ODE) with one variable $\xi$ and two dependent variables $P$ and $Q$. The reduced system is $P_{\xi}=Q_{\xi}=0$.

For the generator $\underline{v}_{2}$, the Boussinesq model is reduced to its steady case.
For the generator $\underline{v}_{3}$, we have the following similarity variables:

$$
\xi=x, \quad P=(1+\zeta) t^{2}, \quad Q=u t .
$$

The reduced system becomes

$$
\begin{aligned}
& Q_{\xi} P+Q P_{\xi}-2 P=0, \\
& Q-Q Q_{\xi}-P_{\xi}-b Q_{\xi \xi}=0 .
\end{aligned}
$$

Case 2: $a \neq 0, b \neq 0$, the trivial case has been covered by case 1 .

Case 3: $a \neq 0, b=0$.

The generator $\underline{v}_{1}$ and $\underline{v}_{2}$ are the same as those in Case 1 .

For the generator $\underline{v}_{3}$, we have the following similarity variables:

$$
\xi=t, \quad P=\zeta, \quad Q=u-\frac{x}{t} .
$$

The reduced ODE becomes

$$
\xi P_{\xi}+1+P=0, \quad \xi Q_{\xi}+Q=0,
$$

which can be solved to give $P=a_{1} / \xi-1$ and $Q=a_{2} / \xi$, where $a_{1}, a_{2}$ are constants.

For the generator $\underline{v}_{4}$, we have the following similarity variables:

$$
\xi=\frac{x}{\sqrt{t}}, \quad P=(1+\zeta) t, \quad Q=u \sqrt{t} .
$$

The reduced system becomes

$$
\begin{aligned}
& 2 P+\xi P_{\xi}-2 Q_{\xi} P-2 Q P_{\xi}+2 a Q_{\xi \xi \xi}=0, \\
& Q_{\xi} \xi+Q-2 Q Q_{\xi}-2 P_{\xi}=0 .
\end{aligned}
$$

\section{SINGLE SOLITARY WAVE SOLUTION}

We search for a solitary wave solution by using the tanh expansion method and a direct integration method.

\section{A. The tanh expansion}

The tanh expansion method is regarded as one of the most straightforward and effective algorithm to obtain solitary wave solutions for a large nonlinear equations. ${ }^{24,25}$ The appeal and success of the method lies in the fact that one circumvents integration to get explicit solutions. For completeness, we give a brief introduction of the method.

Suppose the partial differential equation (PDE) in $u(x, t)$

$$
F\left(u, u_{t}, u_{x}, u_{x x}, u_{x x x}, \ldots\right)=0,
$$

is the system we are studying. In a moving frame of reference, $s=x-\lambda t, u(x, t)$ is a function of $s$ only; the PDE can be transformed into an ordinary differential equation

$$
F\left(u, u_{s}, u_{s s}, \cdots\right)=0 .
$$

We assume that the solution can be expressed as a polynomial of tanh function

$$
u(x, t)=u(s)=\sum_{i=0}^{n} u_{i} \tanh ^{i} k s .
$$


Doing so, we may take advantage of the property that the derivative of $\tanh k s$ is polynomial in $\tanh k s$, i.e., $(\tanh k s)_{s}$ $=k-k \tanh ^{2} k s$. The positive integer $n$ is determined by balancing the highest order linear term with the nonlinear term. Substituting the expansion into the ODE and setting each coefficient of powers of tanh $k s$ to zero, we obtain a system of algebraic equations, from which the parameters $u_{i}$ and wave speed $\lambda$ are explicitly obtained. This is so-called tanh method for traveling wave solution.

Now we apply the tanh method to Boussinesq model, system (d) with $b \neq 0$.

Let

$$
\zeta=\sum_{i=0}^{m} \zeta_{i} f^{i}, \quad u=\sum_{i=0}^{n} u_{i} f^{i}
$$

where $f=f(k s)=f(k(x-\lambda t))$ satisfies

$$
f^{\prime}=1-\frac{k^{2}}{2} f^{2}, \quad \text { or } f=\frac{\sqrt{2}}{k} \tanh \left[\frac{\sqrt{2}}{2} k(x-\lambda t)\right] .
$$

Substituting the expansion into system (d) and balancing the highest order linear term with the nonlinear term, we have $m=n=2$. Collecting the coefficients of $f$ and let them vanish gives

$$
\begin{aligned}
& \zeta_{0}=-\frac{8 b^{2} \lambda^{2} k^{2} a+4 b^{2} \lambda^{2}-a^{2}}{4 b^{2} \lambda^{2}}, \\
& u_{0}=\frac{8 b^{2} \lambda^{2} k^{2}+a+2 b \lambda^{2}}{2 b \lambda}, \\
& \zeta_{1}=0, \quad u_{1}=0 ; \quad \zeta_{2}=\frac{3}{2} a k^{4}, \quad u_{2}=-3 b \lambda k^{4} .
\end{aligned}
$$

The traveling wave solution can be written as

$$
\begin{aligned}
\zeta= & -\frac{8 b^{2} \lambda^{2} k^{2} a+4 b^{2} \lambda^{2}-a^{2}}{4 b^{2} \lambda^{2}} \\
& +3 a k^{2} \tanh ^{2}\left[\frac{\sqrt{2}}{2} k(x-\lambda t)\right] \\
u= & \frac{8 b^{2} \lambda^{2} k^{2}+a+2 b \lambda^{2}}{2 b \lambda}-6 b \lambda k^{2} \tanh ^{2}\left[\frac{\sqrt{2}}{2} k(x-\lambda t)\right] .
\end{aligned}
$$

In order to satisfy the zero boundary conditions at infinity, we have to fix the constants $k$ and $\lambda$ to be

$$
k^{2}=\frac{2 b+a}{4 b a}, \quad \lambda^{2}=\frac{a^{2}}{b(2 b-a)},
$$

and then the solution becomes

$$
\begin{aligned}
& \zeta=-\frac{3(2 b+a)}{4 b} \operatorname{sech}^{2}\left[\frac{\sqrt{2}}{2} k(x-\lambda t)\right], \\
& u=\frac{3 a(2 b+a)}{2 b \lambda(2 b-a)} \operatorname{sech}^{2}\left[\frac{\sqrt{2}}{2} k(x-\lambda t)\right] .
\end{aligned}
$$

For example, for Nwogu's ${ }^{10}$ optimal depth, $\theta=-0.531+1$ $=0.469$,

$$
a=-\frac{1}{2}\left(\theta^{2}-\frac{1}{3}\right)=0.0567, \quad b=\frac{1}{2}\left(1-\theta^{2}\right)=0.3900,
$$

the solitary wave solution is then given by

$$
\begin{aligned}
& \zeta=-1.609 \operatorname{sech}^{2}\left[\frac{\sqrt{2}}{2} k(x-\lambda t)\right], \\
& u=2.363 \operatorname{sech}^{2}\left[\frac{\sqrt{2}}{2} k(x-\lambda t)\right],
\end{aligned}
$$

where $k=3.076$, and $\lambda=0.1067$. The solitary wave solution is not physically meaningful because the wave amplitude is negative. Due to the expansion proposed at the beginning, the solution obtained with the tanh expansion method is very restricted. The solution (44) is not physical in general, but it can be used to test a numerical code developed for the Boussinesq model since it is an analytical solution of the model.

\section{B. Direct integration}

The physically significant solitary wave solution of system (d) can be obtained by setting $\zeta=\zeta(s)$ and $u=u(s)$, where $s=x-\lambda t$ and $\lambda$ is the undermined wave speed. Substituting these relations in the equations in system (d) and integrating the resulting equations once under regularity condition at infinity, we obtain

$$
\begin{aligned}
& -\lambda \zeta+(1+\zeta) u=a u_{s s}, \\
& -\lambda u+\frac{1}{2} u^{2}+\zeta=-b \lambda u_{s s} .
\end{aligned}
$$

Eliminating $u_{s s}$ between these equations yields

$$
\zeta=\frac{(b-a) \lambda u+\frac{1}{2} a u^{2}}{b \lambda^{2}-a-b \lambda u} \equiv Z(u ; \lambda)
$$

Then substituting (47) into (46) leads to the following first integral $(b \neq 0)$,

$$
\begin{aligned}
u_{s}^{2}= & -\frac{1}{3 b \lambda} u^{3}+\left(\frac{1}{b}+\frac{a}{2 b^{2} \lambda^{2}}\right) u^{2} \\
& +\frac{a}{b^{2} \lambda}\left(\frac{2 b}{a}-1-\frac{a}{b \lambda^{2}}\right) \\
& \times\left[u+\left(\lambda-\frac{a}{b \lambda}\right) \ln \left(1-\frac{u}{\lambda-\frac{a}{b \lambda}}\right)\right] \equiv G(u ; \lambda) .
\end{aligned}
$$

$G(u ; \lambda)$ has a double zero as $u \rightarrow 0$, so as to have $u$ fall off exponentially at infinity, and a simple zero at $u=u_{c}$ say, at the wave crest where $\zeta=\alpha$. The wave speed $\lambda=\lambda(\alpha)$ is, therefore, given explicitly by

$$
G\left(u_{c}, \lambda\right)=0 \quad \text { and } Z\left(u_{c}, \lambda\right)=\alpha,
$$

which can be solved to give an asymptotic formula for small $\alpha$

$$
\lambda=1+\frac{1}{2} \alpha-\frac{5 b}{24(b-a)} \alpha^{2}+\frac{(87 a+79 b) b}{720(b-a)^{2}} \alpha^{3}+O\left(\alpha^{4}\right) .
$$

The velocity $u=u(s)$ is then given by quadrature 


$$
s= \pm \int_{u_{c}}^{u} \frac{d u}{\sqrt{G(u ; \lambda)}},
$$

and the wave profile $\zeta=\zeta(s)$ by $(47)$.

For the surface velocity system (c), $a=-1 / 3, b=0$, the single solitary wave solution, worked out by Zhang and $\mathrm{Li},{ }^{16}$ is given explicitly

$$
\begin{aligned}
& \hat{u}(s ; \lambda)=\frac{2\left(\lambda^{2}-1\right)}{\lambda+\cosh 2 \xi}, \\
& \zeta(s ; \lambda)=2\left(\lambda^{2}-1\right) \frac{1+\lambda \cosh 2 \xi}{(\lambda+\cosh 2 \xi)^{2}}, \\
& \xi=\sqrt{\frac{3}{4}\left(\lambda^{2}-1\right) s},
\end{aligned}
$$

or written in velocity potential $\hat{\phi}(s)$, defined by $\hat{u}=\hat{\phi}_{x}$,

$$
\begin{aligned}
& \hat{\phi}(s ; \lambda)=\frac{2}{\sqrt{3}} \ln \frac{\sqrt{\lambda+1}+\sqrt{\lambda-1} \tanh \xi}{\sqrt{\lambda+1}-\sqrt{\lambda-1} \tanh \xi}, \\
& \hat{u}(s)=\phi_{s}, \quad \zeta(s)=\lambda \phi_{s}-\frac{1}{2}\left(\phi_{s}\right)^{2},
\end{aligned}
$$

and the relationship between wave speed $\lambda$ and the wave amplitude $\alpha$ is $\lambda=1+\frac{1}{2} \alpha$, which is the same as that of Korteweg-de Vries (KdV) equation $\zeta_{t}+\zeta_{x}+\frac{3}{2} \zeta \zeta_{x}+\frac{1}{6} \zeta_{x x x}$ $=0$.

We now assume system (c) is exact and treat system (d) as an approximation of system (c). Under this assumption, we look for an approximate solution of system (d) by using the explicit solution of system (c). The velocity at depth $\theta$ ( $\theta=0$ is at the bottom, $\theta=1$ is on the surface) is given by

$$
u=u_{0}-\frac{1}{2} \theta^{2} u_{0 x x},
$$

and applying on the surface gives

$$
\hat{u}=u_{0}-\frac{1}{2} u_{0 x x},
$$

therefore, we have

$$
u=\hat{u}+\frac{1}{2}\left(1-\theta^{2}\right) u_{0 x x}=\hat{u}+\frac{1}{2}\left(1-\theta^{2}\right) \hat{u}_{x x} .
$$

Replacing the first equation of system (d) by (52) and keeping the second equation, we have a new system $\left(b=\frac{1}{2}\left(1-\theta^{2}\right)\right)$

$$
\begin{aligned}
& u=\hat{u}+b \hat{u}_{x x}, \\
& u_{t}+u u_{x}+\zeta_{x}=b u_{x x t},
\end{aligned}
$$

which is regarded as an approximation of system (d). Introducing velocity potential $\phi$, defined by $u=\phi_{x}$, we have the relation between $\zeta$ and $\phi$

$$
\zeta=-\phi_{t}-\frac{1}{2}\left(\phi_{x}\right)^{2}+b \phi_{x x t},
$$

where $\phi$ is taken to be $\hat{\phi}$ as the first approximation and $\hat{\phi}$ $+b \hat{\phi}_{x x}$ as the second approximation, and $\hat{\phi}$ is given by (51).

We now test the accuracy of the two approximations by comparing the wave profile and speed-amplitude relationship with those of system (d) computed by (49), (47), and (50).

We compare the wave profile first. For the first approximate solution (55) with $\phi=\hat{\phi}$, the velocity and wave elevation are given by

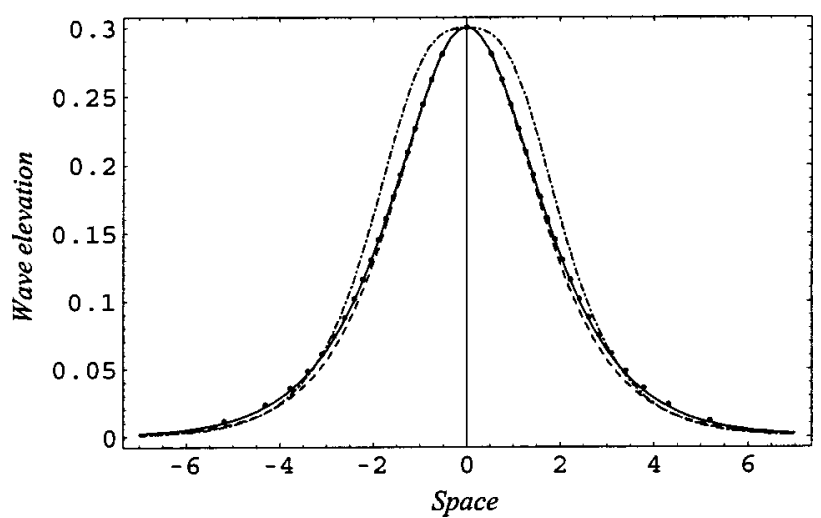

FIG. 1. The comparison on the wave profile between our two approximations and the exact formula for the case $b=1 / 3$. The solid line is the empirical formula of Teng (1997). The dots are exact results of Teng and Wu (1992) generated by a numerical integration. The dashed line is generated by our first approximation. The dash-dot line is generated by our second approximation.

$$
\begin{aligned}
u(s)= & \frac{2\left(\lambda^{2}-1\right)}{\lambda+\cosh 2 \xi}, \quad \xi=\sqrt{\frac{3}{4}\left(\lambda^{2}-1\right)} s, \\
\zeta(s)= & 2\left(\lambda^{2}-1\right)\left[6 b \lambda^{3}-6 b \lambda+\lambda\right. \\
& +\left(3 b \lambda^{4}+(1-3 b) \lambda^{2}+1\right) \cosh 2 \xi \\
& \left.+\left(-3 b \lambda^{3}+3 b \lambda+\lambda\right) \cosh ^{2} 2 \xi\right] /(\lambda+\cosh 2 \xi)^{3} .
\end{aligned}
$$

For the second approximate solution (55) with $\phi=\hat{\phi}$ $+b \hat{\phi}_{x x}$, the velocity is given by

$$
\begin{aligned}
u(s)= & 2\left(\lambda^{2}-1\right)\left[(1-6 b) \lambda^{2}+6 b\right. \\
& +\left(-3 b \lambda^{3}+(2+3 b) \lambda\right) \cosh 2 \xi \\
& \left.+\left(3 b \lambda^{2}+1-3 b\right) \cosh ^{2} 2 \xi\right] /(\lambda+\cosh 2 \xi)^{3} .
\end{aligned}
$$

The formula for the wave elevation is long. It is not presented here for brevity.

In Fig. 1, we present the wave profile for the case of $b$ $=1 / 3$ given by these approximate formulas and the numerically integrations (50) and (47). We also present the empirical formula of Teng ${ }^{26}$

$$
\zeta^{*}(s)=\frac{\alpha \operatorname{sech}^{2} \xi^{*}}{1+\alpha \tanh ^{2} \xi^{*}}, \quad \xi^{*}=\sqrt{\frac{3 \alpha}{4(1+0.68 \alpha)}} s,
$$

which is obtained by a curve fitting with the numerical data. It shows that the first formula (56) gives a good approximate profile compared with the exact numerical results. The first approximation is better than the second one. The result also confirms that Teng's ${ }^{26}$ empirical formula is quite accurate at least for the amplitude shown in the figure.

We now compare the wave speed-amplitude relationship. For the first approximate solution, the wave amplitude can be easily obtained by setting $s=0$ in the wave elevation formula (56). It is written as

$$
\alpha=2(\lambda-1)+6 b(\lambda-1)^{2}+6 b(\lambda-1)^{3},
$$

or

$$
\lambda=1+\frac{1}{2} \alpha-\frac{3}{4} b \alpha^{2}+\frac{3}{8}\left(6 b^{2}-b\right) \alpha^{3}+O\left(\alpha^{4}\right) .
$$




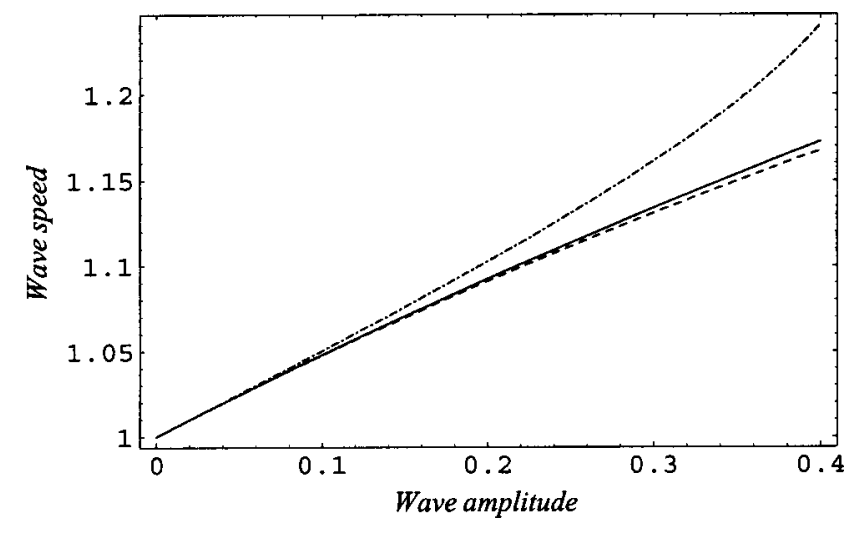

FIG. 2. The comparison on the wave speed-amplitude relationship between our two approximations and the exact formula for the case $b=1 / 3$. The solid line is the exact formula of Teng and Wu (1992). The dashed line is generated by our first approximation. The dash-dot line is generated by our second approximation.

For the second approximate solution (55) with $\phi=\hat{\phi}$ $+b \hat{\phi}_{x x}$, the wave amplitude and wave speed relation is

$$
\begin{aligned}
\alpha= & 2(\lambda-1)+12 b\left(1-6 b^{2}\right)(\lambda-1)^{3} \\
& -72 b^{2}(\lambda-1)^{4}+18 b^{2}(\lambda-1)^{5},
\end{aligned}
$$

or

$$
\lambda=1+\frac{1}{2} \alpha+\frac{3}{4}\left(6 b^{2}-b\right) \alpha^{3}+O\left(\alpha^{4}\right),
$$

where the term with $\alpha^{2}$ vanishes.

For the case of $a=0, b=1 / 3$, system (d) becomes system (a) with depth-mean velocity. The relationship between wave speed $\lambda$ and the wave amplitude $\alpha$ is given by ${ }^{27}$

$$
\lambda=\sqrt{\frac{6(1+\alpha)^{2}}{\alpha^{2}(3+2 \alpha)}[(1+\alpha) \ln (1+\alpha)-\alpha]},
$$

which can be expanded for small $\alpha$,

$$
\lambda=1+\frac{1}{2} \alpha-\frac{5}{24} \alpha^{2}+\frac{79}{720} \alpha^{3}+O\left(\alpha^{4}\right) \text {. }
$$

In Fig. 2, we present the wave speed-amplitude relationship given by the two approximations (57) and (59) and the exact formula (61) for the case of $b=1 / 3$. It shows that the first approximation gives a better result compared with the exact formula of Teng and $\mathrm{Wu} .^{27}$ This can also be observed by a direct comparison between the three asymptotic formulas (58), (60), and (62).

These comparisons suggests that the first approximation $\zeta=-\hat{\phi}_{t}-\frac{1}{2}\left(\hat{\phi}_{x}\right)^{2}+b \hat{\phi}_{x x t}$ gives a good result for both the wave profile and wave speed-amplitude relation. In the next section, this approximation will be adopted to study the multiple solitary wave solution of the Boussinesq model.

\section{APPROXIMATE MULTIPLE SOLITARY WAVE SOLUTION}

The exact multi-soliton solution of system (c) has been provided by Zhang and $\mathrm{Li}^{16}$ As an example, we present twosoliton solution here. The solution for overtaking collision, is written in velocity potential $\hat{\phi}$ as follows:

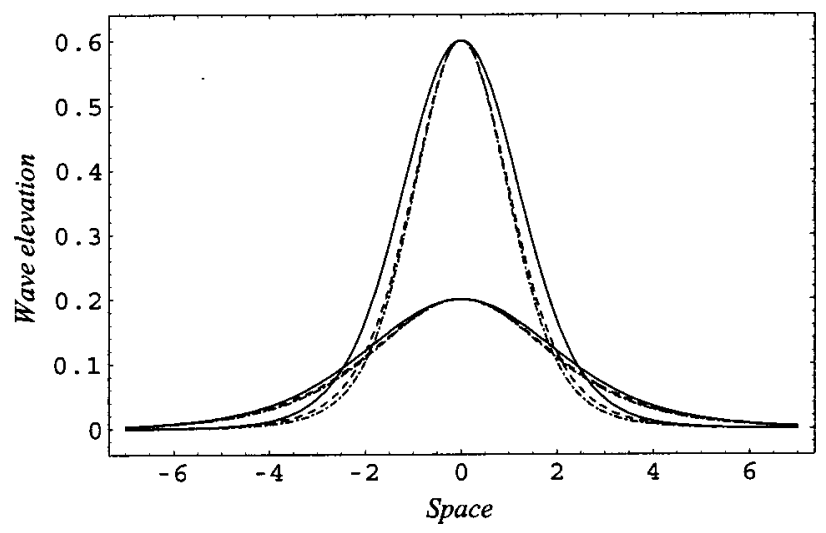

FIG. 3. The two solitary waves with amplitudes $\alpha_{1}=0.2$ and $\alpha_{2}=0.6$ used for the demonstrations of the overtaking and head-on collisions in the next six figures. The solid line is for the surface velocity system. The dashed line is for the depth-mean velocity system, i.e., $b=1 / 3$. The dash-dot line is for the bottom velocity system, i.e., $b=1 / 2$.

$$
\begin{aligned}
\hat{\phi} & =\frac{2}{\sqrt{3}} \ln \frac{k_{2}-k_{1} \tanh \xi_{1} \tanh \xi_{2}+\left(\lambda_{2}-\lambda_{1}\right) \tanh \xi_{2}}{k_{2}-k_{1} \tanh \xi_{1} \tanh \xi_{2}-\left(\lambda_{2}-\lambda_{1}\right) \tanh \xi_{2}}, \\
\xi_{i} & =\frac{\sqrt{3}}{2} k_{i}\left(x-\lambda_{i} t\right), \quad k_{i}=\sqrt{\lambda_{i}^{2}-1}, \\
& i=1,2, \quad \lambda_{2}>\lambda_{1}>1 .
\end{aligned}
$$

The solution for head-on collision is written as

$$
\begin{aligned}
& \hat{\phi}=\frac{2}{\sqrt{3}} \ln \frac{k_{2} \tanh \xi_{2}-k_{1} \tanh \xi_{1}-\left(\lambda_{2}+\lambda_{1}\right)}{k_{2} \tanh \xi_{2}-k_{1} \tanh \xi_{1}+\left(\lambda_{2}+\lambda_{1}\right)}, \\
& \xi_{1}=\frac{\sqrt{3}}{2} k_{1}\left(x-\lambda_{1} t\right), \quad \xi_{2}=\frac{\sqrt{3}}{2} k_{2}\left(x+\lambda_{2} t\right), \\
& k_{i}=\sqrt{\lambda_{i}^{2}-1}, \quad \lambda_{i}>1, \quad i=1,2 .
\end{aligned}
$$

With the velocity potential, we are able to have an approximate solution of the Boussinesq model

$$
u=\hat{\phi}_{x}, \quad \zeta=-\hat{\phi}_{t}-\frac{1}{2}\left(\hat{\phi}_{x}\right)^{2}+b \hat{\phi}_{x x t} .
$$

The solution is exact when $b=0$. If we take $\lambda_{1}=1$, the solution is reduced to a single solitary wave solution.

We now demonstrate the solution graphically. We pick two solitary waves with amplitude $\alpha_{1}=0.2$ and $\alpha_{2}=0.6$ for both overtaking and head-on collisions. The speed of each solitary wave can be determined by solving (57) for the Boussinesq model, and is given by

$$
\begin{aligned}
& b=0, \quad \lambda_{1}=1.1, \quad \lambda_{2}=1.3, \\
& b=\frac{1}{3}, \quad \lambda_{1}=1.09097, \quad \lambda_{2}=1.23304, \\
& b=\frac{1}{2}, \quad \lambda_{1}=1.08751, \quad \lambda_{2}=1.21541 .
\end{aligned}
$$

The wave profiles of the two waves are presented in Fig. 3 for the Boussinesq model. The wave for the surface velocity system is fatter than those for other two systems. The wave for the bottom velocity is the slimmest. 


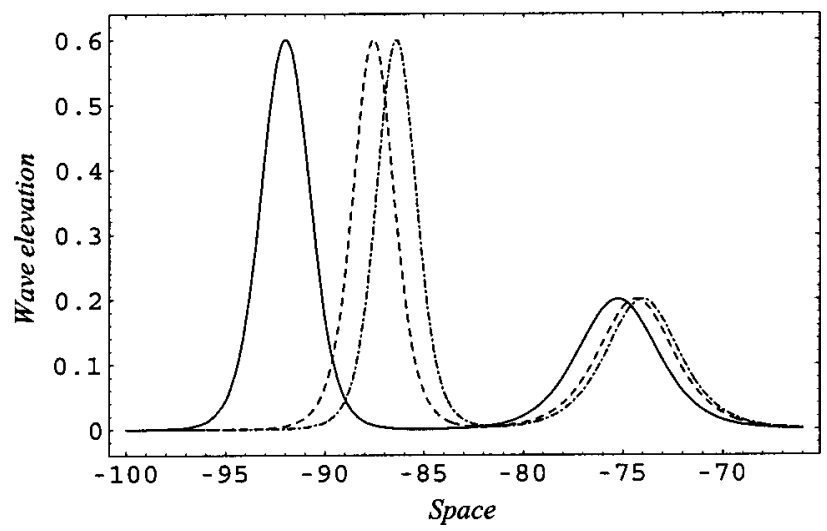

FIG. 4. The snapshot at time $t=-70$ of the overtaking collision of two right-going solitary waves with amplitude $\alpha_{1}=0.2$ and $\alpha_{2}=0.6$. The solid line is for the surface velocity system. The dashed line is for the depth-mean velocity system, i.e., $b=1 / 3$. The dash-dot line is for the bottom velocity system, i.e., $b=1 / 2$.

The overtaking collision of the two solitary wave is shown in Figs. 4-6 for three time instances, $t=-70,0,70$, respectively. At $t=-70$ and 70 , the distance between the solitary waves predicted by three different models is mainly due to their velocity difference. At $t=0$, the wave elevations from three models are very different, which indicates that the correction term $b \hat{u}_{x t}$ plays an important role in the strong interaction. The difference of the local behavior near $t=0$ predicted by the three models is not negligible.

The head-on collision of the two solitary wave is shown in Figs. 7-9 for three time instances, $t=-20,0,20$, respectively. The difference predicted by three models is small in the weak interaction.

\section{DISCUSSION ON INTEGRABILITY}

Integrable nonlinear partial differential equations play very important roles in modeling water wave problem.

Being a unidirectional weakly nonlinear and weakly dispersive water wave model, the $\mathrm{KdV}$ equation

$$
\zeta_{t}+\zeta_{x}+\frac{3}{2} \zeta \zeta_{x}+\frac{1}{6} \zeta_{x x x}=0
$$

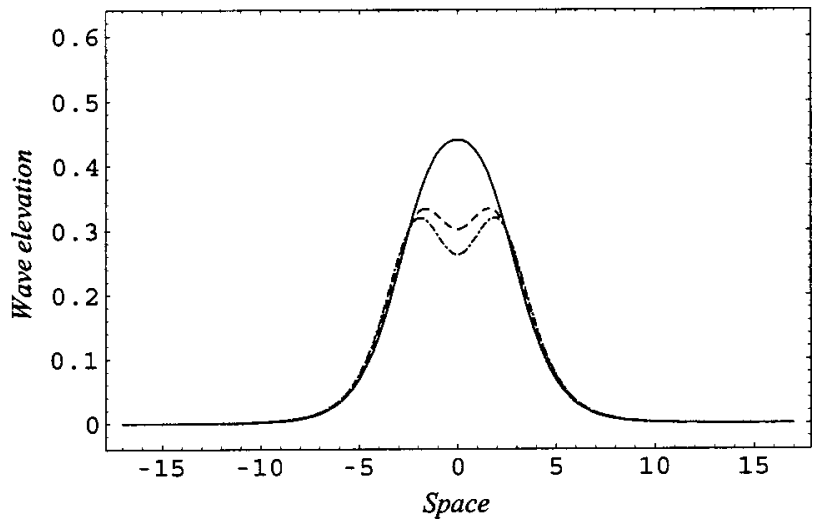

FIG. 5. The snapshot at time $t=0$ of the overtaking collision of two rightgoing solitary waves with amplitude $\alpha_{1}=0.2$ and $\alpha_{2}=0.6$. The solid line is for the surface velocity system. The dashed line is for the depth-mean velocity system, i.e., $b=1 / 3$. The dash-dot line is for the bottom velocity system, i.e., $b=1 / 2$.

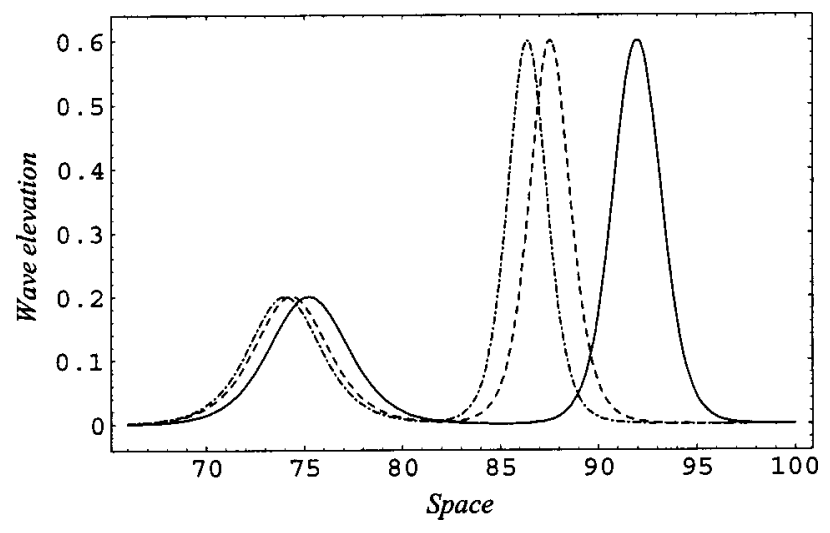

FIG. 6. The snapshot at time $t=70$ of the overtaking collision of two rightgoing solitary waves with amplitude $\alpha_{1}=0.2$ and $\alpha_{2}=0.6$. The solid line is for the surface velocity system. The dashed line is for the depth-mean velocity system, i.e., $b=1 / 3$. The dash-dot line is for the bottom velocity system, i.e., $b=1 / 2$.

is known to be integrable. Modifying the dispersive term without changing its order of magnitude yields the Benjamin-Bona-Mahoney (BBM $)^{28}$ equation

$$
\zeta_{t}+\zeta_{x}+\frac{3}{2} \zeta \zeta_{x}-\frac{1}{6} \zeta_{x x t}=0,
$$

which is not integrable. By including some nonlinear dispersive terms, the BBM equation can be made integrable

$$
\zeta_{t}+\zeta_{x}+\frac{3}{2} \zeta \zeta_{x}-\frac{1}{6} \zeta_{x x t}-\frac{1}{12}\left(\zeta \zeta_{x x}+\frac{1}{2} \zeta_{x}^{2}\right)_{x}=0,
$$

which is known to be Camassa-Holm $(\mathrm{CH})^{29}$ equation.

For a bidirectional water wave model, the classical Boussinesq equation, i.e., system (c), is integrable, but the Boussinesq model with $b \neq 0$ is not. Motivated by the relationship between the $\mathrm{BBM}$ and the $\mathrm{CH}$ equations, we try to add some nonlinear dispersive terms to the Boussinesq model and see whether it can be made integrable. We have done a Painlevé test against the following system:

$$
\zeta_{t}+[(1+\zeta) u]_{x}=a u_{x x x}+a_{1} u_{x} u_{x x}+a_{2} u u_{x x x},
$$

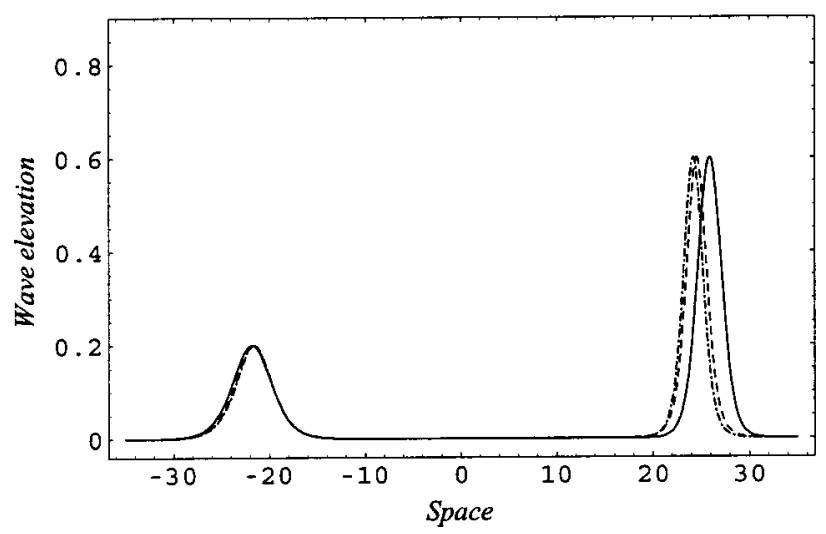

FIG. 7. The snapshot at time $t=-20$ of the head-on collision of two solitary waves with amplitude $\alpha_{1}=0.2$ going to the right and $\alpha_{2}=0.6$ going to the left. The solid line is for the surface velocity system. The dashed line is for the depth-mean velocity system, i.e., $b=1 / 3$. The dash-dot line is for the bottom velocity system, i.e., $b=1 / 2$. 


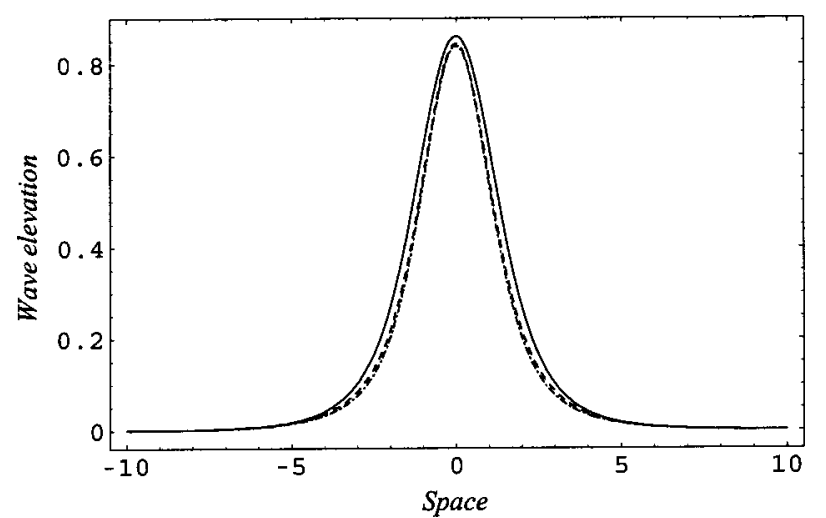

FIG. 8. The snapshot at time $t=0$ of the head-on collision of two solitary waves with amplitude $\alpha_{1}=0.2$ going to the right and $\alpha_{2}=0.6$ going to the left. The solid line is for the surface velocity system. The dashed line is for the depth-mean velocity system, i.e., $b=1 / 3$. The dash-dot line is for the bottom velocity system, i.e., $b=1 / 2$.

$$
u_{t}+u u_{x}+\zeta_{x}=b u_{x x t}+b_{1} u_{x} u_{x x}+b_{2} u u_{x x x},
$$

and cannot find a suitable set of coefficients $a, a_{1}, a_{2}, b$ $\neq 0, b_{1}$, and $b_{2}$, which makes the system Painlevé integrable.

However, applying a simple transformation $\zeta \rightarrow \zeta-b u_{x t}$ to system (c) yields a modified Boussinesq model

$$
\begin{aligned}
& \zeta_{t}+[(1+\zeta) u]_{x}=-\frac{1}{3} u_{x x x}+b u_{x t t}+b\left(u u_{x t}\right)_{x}, \\
& u_{t}+u u_{x}+\zeta_{x}=b u_{x x t},
\end{aligned}
$$

which is integrable because it is equivalent to system (c). This new integrable system is more or less like a bidirectional extension of the Camassa-Holm $(\mathrm{CH})$ equation. As a matter of fact, the solutions we present in the last section are the exact solutions of this new system. The arbitrary parameter $b$ in the system gives us a freedom to match the exact solution with the numerical solution of the full water wave problem modeled with Euler equation, so that we can have the best prediction for the collision of solitary waves especially the strong interaction.

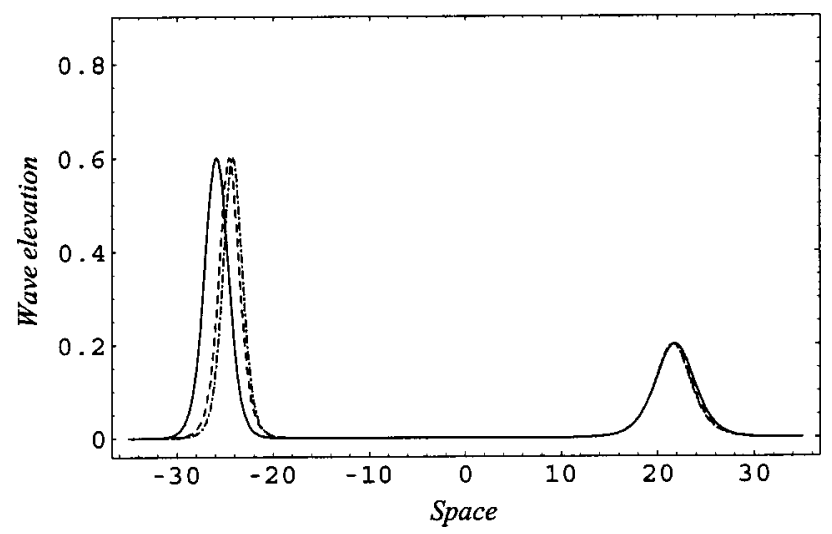

FIG. 9. The snapshot at time $t=20$ of the head-on collision of two solitary waves with amplitude $\alpha_{1}=0.2$ going to the right and $\alpha_{2}=0.6$ going to the left. The solid line is for the surface velocity system. The dashed line is for the depth-mean velocity system, i.e., $b=1 / 3$. The dash-dot line is for the bottom velocity system, i.e., $b=1 / 2$.

\section{CONCLUSION}

We study the mathematical properties of Boussinesq models with constant depth for weakly nonlinear weakly dispersive water waves. The $1+1$ dimensional leading order Boussinesq model is shown to be not Painlevé-integrable. The Lie symmetries and similarity reductions of the Boussinesq model are presented. We also study the solitary wave solutions of the Boussinesq model and propose an approximate single and multiple solitary wave solution based on the exact multi-soliton solutions of Zhang and $\mathrm{Li}^{16}$ developed for the surface-velocity system. The approximate solutions are essentially exact solutions of a modified Boussinesq model, which is integrable due to its equivalency with the surfacevelocity system. A nice feature of the integrable modified Boussinesq model is that it has a free parameter. This allows us to match the exact multiple solitary wave solution with the numerical result of the Euler equation, so that we can have a good prediction of the interaction.

The similar study on the $2+1$ dimensional higher order Boussinesq model is technically more demanding and, therefore, left for further research.

\section{ACKNOWLEDGMENTS}

The authors acknowledge helpful comments and suggestions from an anonymous referee. J.E.Z. has been supported by the Research Grants Council of Hong Kong. Y.S.L. has been supported by the National Natural Science Foundation of China (1030).

${ }^{1}$ D. H. Peregrine, "Long waves on beach," J. Fluid Mech. 27, 815 (1967).

${ }^{2}$ T. Y. Wu, "Long waves in ocean and coastal waves," J. Eng. Mech. 107, 501 (1981).

${ }^{3}$ T. Y. Wu, "Generation of upstream-advancing solitons by moving disturbances," J. Fluid Mech. 184, 75 (1987).

${ }^{4}$ K.-H. Wang, T. Y. Wu, and G. T. Yates, “Three-dimensional scattering of solitary waves by vertical cylinder," J. Waterw., Port, Coastal, Ocean Eng. 118, 551 (1992).

${ }^{5}$ K.-H. Wang, "Diffraction of solitary waves by breakwaters," J. Waterw., Port, Coastal, Ocean Eng. 119, 49 (1993).

${ }^{6}$ A.-M. Shi, M. H. Teng, and T. Y. Wu, "Propagation of solitary waves through significantly curved shallow water channels," J. Fluid Mech. 362, 157 (1998).

${ }^{7}$ J. E. Zhang, I. Run-up of ocean waves on beaches, II. Nonlinear waves in a fluid-filled elastic tube, Ph.D. thesis, California Institute of Technology, Pasadena, 1996.

${ }^{8}$ J. E. Zhang and T. Y. Wu, "Oblique long waves on beach and induced longshore current," J. Eng. Mech. 125, 812 (1999).

${ }^{9}$ J. E. Zhang, T. Y. Wu, and T. Y. Hou, "Coastal hydrodynamics of ocean waves on beach," Adv. Appl. Mech. 37, 89 (2001).

${ }^{10} \mathrm{O}$. Nwogu, "An alternative form of the Boussinesq equations for nearshore wave propagation," J. Waterw., Port, Coastal, Ocean Eng. 119, 618 (1993).

${ }^{11}$ G. Wei and J. T. Kirby, "A time-dependent numerical code for extended Boussinesq equations," J. Waterw., Port, Coastal, Ocean Eng. 120, 251 (1995).

${ }^{12}$ G. Wei, J. T. Kirby, S. T. Grilli, and R. Subramanya, "A fully nonlinear Boussinesq model for surface waves. I. Highly nonlinear unsteady waves," J. Fluid Mech. 294, 71 (1995).

${ }^{13}$ T. Y. Wu and J. E. Zhang, "On modeling nonlinear long waves," in Mathematics Is for Solving Problems, edited by L. P. Cook, V. Roytburd, and M. Tulin (SIAM, Philadelphia, 1996), p. 233.

${ }^{14}$ C.-L. Chen, X.-Y. Tang, and S.-Y. Lou, "Solution of a (2+1)-dimensional dispersive long wave equation,” Phys. Rev. E 66, 036605 (2002).

${ }^{15}$ X.-D. Ji, C.-L. Chen, J. E. Zhang, and Y.-S. Li, "Lie symmetry analysis 
and some new exact solutions of the Wu-Zhang equation," J. Math. Phys. 45, 448 (2004)

${ }^{16}$ J. E. Zhang and Y.-S. Li, "Bidirectional solitons on water," Phys. Rev. E 67, 016306 (2003).

${ }^{17}$ Y.-S. Li, W.-X. Ma, and J. E. Zhang, "Darboux transformations of classical Boussinesq system and its new solutions," Phys. Lett. A 275, 60 (2000).

${ }^{18}$ Y.-S. Li and J. E. Zhang, "Darboux transformations of classical Boussinesq system and its multi-soliton solutions," Phys. Lett. A 284, 253 (2001).

${ }^{19}$ Y.-S. Li and J. E. Zhang, "Bidirectional soliton solutions of the classical Boussinesq system and AKNS system," Chaos, Solitons Fractals 16, 271 (2003).

${ }^{20}$ J. E. Zhang and Y.-S. Li, "Theory of bidirectional solitons on water," in Proceedings of Nonlinear Evolution Equations and Dynamical System, ICM 2002 Satellite Conference, edited by Y. Cheng, S. Hu, Y.-S. Li, and C.-K. Pen (World Scientific, Singapore, 2003), p. 135.

${ }^{21}$ J. Weiss, M. Tabor, and G. Carnevale, "The Painlevé property for partial differential equations," J. Math. Phys. 24, 522 (1983).
${ }^{22}$ R. L. Sachs, "On the integrable variant of the Boussinesq system: Painlevé property, rational solutions, a related many-body system, and equivalence with the AKNS hierarchy," Physica D 30, 1 (1988).

${ }^{23}$ P. J. Olver, Applications of Lie Groups to Differential Equations, 2nd ed. (Springer-Verlag, New York, 1993).

${ }^{24}$ W. Malfliet, "Solitary wave solutions of nonlinear-wave equations," Am. J. Phys. 60, 650 (1992).

${ }^{25}$ W. Malfliet and W. Hereman, "The tanh method 1. Exact solutions of nonlinear evolution and wave equations," Phys. Scr. 54, 563 (1996).

${ }^{26}$ M. H. Teng, "Solitary wave solution to Boussinesq equations," J. Waterw., Port, Coastal, Ocean Eng. 123, 138 (1997).

${ }^{27}$ M. H. Teng and T. Y. Wu, "Nonlinear water waves in channels of arbitrary shape," J. Fluid Mech. 242, 211 (1992).

${ }^{28}$ T. B. Benjamin, J. L. Bona, and J. J. Mahoney, "Model equations for long waves in nonlinear dispersive systems," Philos. Trans. R. Soc. London, Ser. A 227, 47 (1972)

${ }^{29}$ R. Camassa and D. Holm, "A new integrable shallow water equation," Phys. Rev. Lett. 71, 1661 (1993). 